\title{
Death certification: issues and interventions
}

\author{
Dan Middleton $^{1^{\star}}$, Robert Anderson ${ }^{2}$, Tiffini Billingsly ${ }^{3}$, Nombulelo Bande Mangaliso Virgil ${ }^{3}$, \\ Yolanda Wimberly ${ }^{3}$, Robin Lee ${ }^{1}$
}

\footnotetext{
${ }^{1}$ Health Investigations Branch, Division of Health Studies, Agency for Toxic Substances and Disease Registry, Atlanta, USA;

*Corresponding Author: dcm2@cdc.gov

${ }^{2}$ National Center for Health Statistics, Center for Disease Control and Prevention, Atlanta, USA;

${ }^{3}$ Morehouse School of Medicine, Atlanta, USA.
}

Received 21 August 2011; revised 7 October 2011; accepted 27 October 2011.

\begin{abstract}
A review of the literature suggests that errors in death certification are common. We reviewed the published literature to clarify what is known and what remains to be learned before evidencebased changes in medical education can be recommended. We searched the National Library of Medicine's PubMed database for articles that addressed death certificate accuracy and identified 159 articles of interest published from 1996 to 2010. Among these 159 articles, we found 83 that were relevant to our goals and objectives. Cause of death certification has been shown to be problematic and several interventions have been shown to improve its accuracy, especially if the intervention is interactive. However these studies have focused on short term gains rather than on long term retention and performance, leaving a significant data gap. We suggest a study design that could address this data gap.
\end{abstract}

Keywords: Cause of Death; Death Certification

\section{INTRODUCTION}

\subsection{Background}

Death certificates contain a medical section that is usually completed by the attending physician, or in special cases by a medical examiner or coroner. A nosologist then codes the "cause of death" (COD) information for statistical purposes. The coded data can then be used to rank causes of death among specific groups [1] and combined with other data to identify exposures associated with fatal diseases, assess diagnostic testing, and determine the risk and effectiveness of therapeutic techniques. The accuracy of such analyses depends largely on the raw COD data that physicians record on individ- ual death certificates.

\subsection{Model Death Certificates}

The Centers for Disease Control and Prevention's National Center for Health Statistics (NCHS) provides a two part standard certificate of death as a model for States to follow [1]. The first part is for reporting the chain of events that lead directly to the death. The COD listed in this section elicits the certifying physician's opinion on the decedents' underlying COD, defined as "the disease or injury which initiated the train of morbid events leading directly to death, or the circumstances of the accident or violence which produced the fatal injury.”

The second part of the standard certificate contains information on all other diseases, conditions, or injuries that contributed to death. These are commonly referred to as the multiple causes or contributing CODs.

This content and format of the standard certificate is followed by each U.S. state with only minor variations. This provides uniform data across the States that can be compared and aggregated for national statistics. While the standard certificate has promoted consistency in the kinds of data collected, it has not resolved the issues associated with ensuring accurate COD information.

\subsection{Goals and Objectives}

We propose to review what is known about the quality of COD data from death certificates, including:

a) overall accuracy;

b) physicians' awareness of death certificate uses and importance; and

c) educational interventions designed to improve death certification and COD data.

After summarizing these key issues according to current knowledge, we describe the "next steps" for making progress in the accuracy of death certificates. These 
"next steps" are vital for public health progress.

\section{METHODS}

We searched the National Library of Medicine's PubMed database for articles published in English between January 1996 and August 2010. The search term used was "death certificate accuracy". We also explored the references cited by these articles.

\section{FINDINGS}

\subsection{Summary}

We found 154 articles in the PubMed search and 5 additional articles of interest among the references. Among these 159 articles, we identified 83 that were relevant to our goals and objectives. Four articles addressed physician attitudes. The remaining 79 articles focused on accuracy ( $n=71$ ), educational interventions $(n=7)$, or both $(n=1)$. We used this literature to provide examples and citations for salient points.

\subsection{Death Certificate Accuracy}

Generally speaking, accuracy concerns relate to either listing an incorrect COD or omitting important information. In 2001, Smith Sehdev and Hutchins [2] compared clinical and autopsy COD statements for 494 cases between June 1995 and February 1997 at Johns Hopkins Medical Institutions [2]. They compared the CODs for accuracy based on guidelines created by the College of American Pathologists and the NCHS. The authors reported that $41 \%$ of the death certificates contained improperly completed COD statements; $24 \%$ had major discrepancies between the clinicians' and the pathologists' COD. The authors concluded that the COD statements on death certificates were not reliable sources of information for national mortality statistics.

Using mock death certificates, Lakkireddy et al. [3] evaluated the COD statements completed by 590 residents from various training programs nationwide. For a sample case of in-hospital death due to urosepsis, they found $45 \%$ of the residents incorrectly identified a cardiovascular event as the primary COD.

Selikoff and Seidman [4] looked at asbestos-related diseases using the "best evidence" for 17,800 asbestos insulation workers in the United States and Canada from 1967-1986. "Best evidence" was defined to include histopathology, autopsy and medical records. They found that $11.9 \%$ of lung cancers related to asbestos and $38 \%$ of mesotheliomas would have been missed had their assessment been limited to only death certificate data. There was also discordance between death certificates and best evidence for asbestos-related kidney cancer.
Pritt et al. [5] reviewed 50 clinical summaries for patients treated at the University of Vermont between January 2002 and December 2003. Mock death certificates were created using the information available and then compared to the originals. They found that $34 \%$ of the original death certificates had omissions or listed the wrong COD or manner of death.

Data from the Framingham Heart Study indicate that coronary heart disease as a COD may have been overestimated. Death certificates attributed 24\% more deaths to coronary heart disease (overall) than did a panel of three physicians. For persons at least 85 years of age, death certificates attributed more than twice as many deaths to coronary heart disease than did the physician panel [6].

Problems with death certification are not limited to the United States. Nielsen et al. [7] compared the death certificates and autopsy results for 433 hospital patients at the University of Iceland. They found significant discrepancies between the two documents in 50\% of these patients. The immediate COD was incorrect on $25 \%$ of the certificates. D'Amico et al. [8] investigated the extent of misclassification of the underlying COD attributed to ill-defined and/or multiple causes in Naples, Italy. They found a discordance of approximately $54 \%$ for both ill-defined and multiple COD when comparing the initial disease coding to the coding provided after interviewing the certifying physician and reviewing the medical records of deceased patients.

In addition to problems with accuracy, certifying physicians often do not understand how to properly report COD in the standard format. Two common problems in the first part of COD certification are reporting multiple conditions per line (contrary to the instructions) and reporting illogical causal sequences. For example, Lu et al. [9] note that hypertension and acute myocardial infarcttion are often reported as the cause of diabetes. A look at detailed mortality data for 2007 from the National Vital Statistics System (data available from: http://www.cdc. gov/nchs/deaths.htm) shows that illogical sequences appear on $30 \%$ of all U.S. death certificates and multiple conditions are reported on the same line in the first part of COD certification for $9 \%$ of all deaths.

\subsection{Physician Awareness}

One factor that potentially limits physician performance is their awareness of the importance of death certificates and how they are used. While the literature concerning physicians' awareness is sparse, researchers have questioned whether physicians are aware of the public health importance of death certificate data $[2,10,11]$.

Degani et al. [10] asked 123 third year medical students at Mercer University School of Medicine to identify what is significant about death certificates. Prior to 
an intervention, 36\% of respondents listed legal importance and 23\% listed statistical importance. Post-intervention, the proportion who listed legal importance dropped to $19 \%$, while the proportion listing statistical importance rose to $44 \%$. Other purposes that were suggested included research, documentation, insurance, and decedents' families as significant reasons for completing the death certificate.

\subsection{Educational Interventions}

Given the widespread inaccuracies, various intervenetions have been attempted to improve the accuracy of death certificates. Aung et al. [12] provide an overview of various interventions published between 1989 and 2007. For example, Lakkireddy et al. [13] studied internal medicine residents. Death certificates were scored using the Mid America Heart Institute (MAHI) Death Certificate Scoring System. The MAHI system was based on guidelines from the College of American Pathologists, the National Association of Medical Examiners, and the NCHS. Two hundred residents were divided into an interactive workshop group and a printed educational materials group. After the interventions, the COD score in the workshop group increased from $15 \%$ to $91 \%$ and the COD score in the printed materials group increased from $16 \%$ to $55 \%$. The researchers concluded that an educational intervention improved medical residents' accuracy in completing COD statements. More specifically, the interactive workshop yielded better results than did the more passive printed educational materials.

Villar and Perez-Mendez [14] performed a study in Spain over 18 months in which 166 medical trainees from various specialties attended a 90-minute seminar on completing death certificates. Prior to the intervention $71 \%$ of the certificates had errors. The most common error was listing a mechanism of death instead of the cause. After the intervention the error rate was reduced to $9 \%$; none of the participants listed a mechanism of death after the intervention. The authors concluded that a simple educational intervention can dramatically improve the accuracy of death certificates completed by physicians.

\section{DISCUSSION}

The common shortcomings of COD information on death certificates have been well-documented. Currently, it appears that few medical schools or residency training programs provide formal training in death certification, relying on a vague expectation that the topic will be covered "on the job” in hospital wards or during intensive care rotations.
While nosologists use an established system of rules to code deaths not stated clearly and properly, these corrective procedures do not always result in accurate coding [15]. At times the certifier must be contacted for clarification; while this may improve the coding, it is likely to be time consuming.

In general, studies of death certificate accuracy have compared the COD recorded on the death certificate to one generated by an expert panel of physicians who reviewed the medical record and/or autopsy findings. We note that such comparisons could overestimate the frequency of inaccuracies. For example, medical records are often incomplete and tend to be more oriented to the reason for hospital admission and treatment. They do not always contain information that elicits the appropriate underlying COD. Similarly, autopsy reports tend to focus on the immediate COD rather than the underlying COD. Overall, these reports can be used for comparison, but should not be assumed to represent a true "gold standard” [16].

Regardless, COD certification is known to be problematic and several interventions have been shown to improve its accuracy, especially if the intervention is interactive. However these studies have focused on short term gains and not on long term retention and performance, creating a significant data gap in what is known about the value of educational interventions. To address this gap, we recommend an interventional study (or studies) to resolve this issue.

A group of physicians-in-training would undergo pre-intervention testing, an educational intervention, and post-intervention testing. In contrast to previous studies, the physicians-in-training would also be retested after a longer period of time (e.g. one year later) to assess their long term retention and performance. Careful study design and analyses will allow researchers to assess the intervention's short term effectiveness and the long term retention of key concepts and performance one or more years after the educational intervention.

Generally speaking, changes in medical training should be evidence-based. Information on the long term retention described above is needed to overcome the inertia that prevents the allocation of time and resources for death certification training in medical residency programs. By clarifying this remaining data gap, we hope to stimulate research that will lead to improved death certification and more accurate vital statistics.

\section{REFERENCES}

[1] CDC (2003) Physicians handbook on medical certification of death. U.S. Department of Health and Human Services, Centers for Disease Control and Prevention (CDC). 
Publication No. 2003-1108, Hyattsville.

[2] Smith-Sehdev, A.E. and Hutchins, G.M. (2001) Problems with proper completion and accuracy of the causeof-death statement. Archives of Internal Medicine, 161, 277-284. doi:10.1001/archinte.161.2.277

[3] Lakkireddy, D.R., Gowda, M.S., Murray, C.W., Basarakodu, .K.R. and Vacek, J.L. (2004) Death certificate completion: How well are physicians trained and are cardiovascular causes overstated? American Journal of Medicine, 117, 492-498. doi:10.1016/j.amjmed.2004.04.018

[4] Selikoff, I.J. and Seidman, H. (1992) Use of death certificates in epidemiological studies, including occupational hazards: Variations in discordance of different asbestos-associated diseases on best evidence ascertainment. American Journal of Industrial Medicine, 22, 481492. doi:10.1002/ajim.4700220403

[5] Pritt, B.S., Hardin, N.J., Richmond, J.A. and Shapiro, S.L. (2005) Death certification errors at an academic institution. Archives of Pathology and Laboratory Medicine, 129, 1476-1479.

[6] Lloyd-Jones, D.M., Martin, D.O., Larson, M.G. and, Levy, D. (1998) Accuracy of death certificates for coding coronary heart disease as the cause of death. Annals of Internal Medicine, 129, 1020-1026.

[7] Nielsen, G.P., Bjornsson, J. and Jonasson, J.G. (1991) The accuracy of death certificates. Implications for health statistics. Virchows Archiv. A, Pathological Anatomy and Histopathology, 419, 143-146. doi:10.1007/BF01600228

[8] D'Amico, M., Agozzino, E., Biagino, A., Simonetti, A. and Marinelli, P. (1999) Ill-defined and multiple causes on death certificates - a study of misclassification in mortality statistics. European Journal of Epidemiology, 15, 141-148. doi:10.1023/A:1007570405888

[9] Lu, T.H., Hsu, P.Y., Bjorkenstam, C. and Anderson, R.N.
(2006) Certifying diabetes-related cause-of-death: A comparison of inappropriate certification statements in Sweden, Taiwan and the USA. Diabetologia, 49, 28782881. doi:10.1007/s00125-006-0470-6

[10] Degani, A.T., Patel, R.M., Smith, B.E. and Grimsley, E. (2009) The effect of student training on accuracy of completion of death certificates. Medical Education Online, 14, 17. doi:10.3885/meo.2009.Res00315

[11] O’Donovan, B.G., Armstrong, P., Byrne, M.C. and Murphy, A.W. (2010) A mixed-methods prospective study of death certification in general practice. Family Practice, 27, 351-355. doi:10.1093/fampra/cmq012

[12] Aung, E., Rao, C. and Walker, S. (2010) Teaching causeof-death certification: Lessons from international experience. Postgraduate Medical Journal, 86, 143-152. doi:10.1136/pgmj.2009.089821

[13] Lakkireddy, D.R., Basarakodu, K.R., Vacek, J.L., Kondur, A.K., Ramachandruni, S.K., Esterbrooks, D.J., et al. (2007) Improving death certificate completion: A trial of two training interventions. Journal of General Internal Medicine, 22, 544-548. doi:10.1007/s11606-006-0071-6

[14] Villar, J. and Perez-Mendez, L. (2007) Evaluating an educational intervention to improve the accuracy of death certification among trainees from various specialties. BMC Health Services Research, 7, 183. doi:10.1186/1472-6963-7-183

[15] Hanzlick, R. (2006) Cause of Death and the Death Certificate. College of American Pathologists. Northfield, Illinois, 235.

[16] Johansson, L.A., Westerling, R. and Rosenberg, H.M. (2006) Methodology of studies evaluating death certificate accuracy were flawed. Journal of Clinical Epidemiology, 59, 125-131. doi:10.1016/j.jclinepi.2005.05.006 